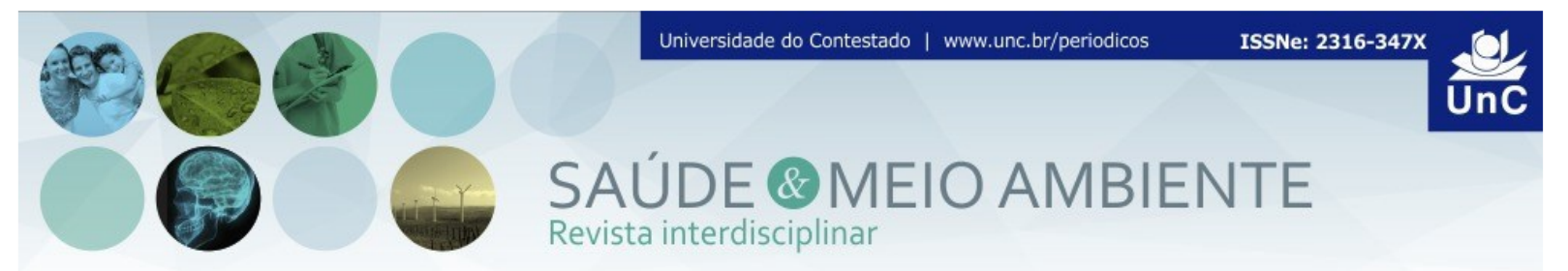

\title{
AVALIAÇÃO E CARACTERIZAÇÃO DE INSALUBRIDADE PRO EXPOSIÇÃO Á RUÍDO EM UMA EMPRESA DE RECICLAGEM DE PLÁSTICO
}

\author{
Roger Francisco Ferreira de Campos ${ }^{1}$ \\ Antonio Pedro Tessaro ${ }^{2}$
}

\begin{abstract}
RESUMO
As condições de trabalho em muitas empresas e indústrias possibilitam situações de risco à qualidade de vida dos seus colaboradores, dentre essas situações está à exposição aos altos níveis de ruído. Portanto, o presente trabalho tem como objetivo avaliar a higiene ocupacional de um colaborador no processo de extrusão de plástico em uma empresa de reciclagem, através da análise da emissão de ruído, com a perspectiva do cálculo de insalubridade. O trabalho foi realizado em uma empresa de recuperação de plástico do município de Caçador/SC, para os cálculos de insalubridade seguiu a partir da dose e exposição ao ruído conforme a NR-15 e NHO-01. Através do estudo pode-se observar que o colaborador da empresa está sob uma situação de exposição inaceitável, obtendo a consideração técnica como critica e nível de atuação recomendada para ações de controle urgente, conformes as diretrizes de cálculos da NR-15 e NHO-01, visto que o nível do ruído Leq é de $87,18 \mathrm{~dB}(\mathrm{~A})$, buscando atenuar esse questão o colaborador recebe Equipamento de Proteção Individual (EPI) que atenua o ruído. Assim, é preciso de um monitoramento continuo da segurança do trabalhador em questão, visto que se o mesmo não realizar a utilização do EPI o mesmo pode sofrer consequências no seu processo de audição.
\end{abstract}

Palavras-chave: Monitoramento do Ruído. Ruído Ocupacional. Ruído.

\section{EVALUATION AND CHARACTERIZATION OF UNSAFE BY NOISE EXPOSURE IN A PLASTIC RECYCLING}

\begin{abstract}
The working conditions in many companies and industries allow situations of risk to the quality of life of its employees; among these situations is the exposure to high noise levels. Therefore, the present work has the objective of evaluating the occupational hygiene of a plasterer in the plastic extrusion process in a plastic recycling company, through the analysis of the noise emission, with the perspective

\footnotetext{
${ }^{1}$ Engenheiro Ambiental, Especialista em Segurança do Trabalho, Mestre em Ciências Ambientais pela Universidade Estadual de Santa Catarina - UDESC/CAV. Professor da Universidade Alto Vale do Rio do Peixe (UNIARP). Caçador. Santa Catarina. Brasil. E-mail: roger@uniarp.edu.br.

2Engenheiro Elétrico, Especialista em Segurança do Trabalho, Mestre em Engenharia: Energia, Ambiente e Materiais pela Universidade Luterana do Brasil - ULBRA. Professor da Universidade Alto Vale do Rio do Peixe (UNIARP). Caçador. Santa Catarina. Brasil. E-mail: tessaro@uniap.edu.br
}

Saúde Meio Ambient. v. 8, p. 137-147, 2019

ISSNe 2316-347X 
of the calculation of insalubrity. The work was carried out in a plastic recovery company of the municipality of Caçador/SC, for calculations of unhealthiness followed from the dose and exposure to noise according to NR-15 and NHO-01. Through the study it can be observed that the employee of the company is on an unacceptable exposure situation, obtaining the technical consideration as critical and recommended action level for actions of urgent control, according to calculations made by NR-15 and NHO-01, seen that the Leq noise level is $87.18 \mathrm{~dB}(\mathrm{~A})$, seeking to attenuate this issue the employee receives Individual Protection Equipment (IPE) that attenuates noise. Thus, continuous monitoring of the safety of the worker in question is required, since if it does not make the use of IPE possible, it can have consequences in the hearing process.

Key words: Noise Measurement. Noise Monitoring. Noise Occupational.

\section{INTRODUÇÃO}

Com o desenvolvimento tecnológico, desencadeia-se principalmente o aumento dos processos produtivos, como também a mecanização de computadores e equipamentos. Com isso, o homem enquanto um ser reflexivo, produtivo, pensante e construtor de seu mundo, deve buscar alternativas viáveis e corretas para a aplicação dos recursos naturais, para a exposição a agentes nocivos à saúde, para a convivência humana e principalmente para a sua qualidade de vida - mediante ao processo tecnológico (COELHO; COELHO, 2015).

Segundo Braga et al., (2005) entre os agentes nocivos à saúde, pode-se destacar o agente físico "ruído", determinado como uma vibração acústica capaz de produzir sensação auditiva - sendo sua intensidade feita em decibéis (dB). O ruído é conhecido como uma poluição, associado ao ruído estridente ou som não desejado, sendo estabelecida pela pressão exercida no sistema auditivo, portanto, quando essa pressão provoca danos à saúde humana, sendo ela comportamental ou física, esse agente deve ser tratado como uma poluição.

O corpo humano sobre grandes níveis de pressão sonora (ruído) pode sofrer com o zumbido, insônia, estresse, irritabilidade, aumento da pressão arterial e da frequência cardíaca (LUSK et al., 2002) e perda auditiva (ARAUJO, 2002). Obtendo como consequência mais grave à perda auditiva, a qual está relacionada com as doenças do trabalho, caracterizada como Perda Auditiva Induzida por Ruído Ocupacional - PAIRO (SILVA; MENDES, 2005), sendo que grande parte dos processos produtivos e industriais possui a emissão de ruído (MELLO; SANTOS, 2014).

Uma das medidas de prevenção dos efeitos causados pelo ruído é a utilização de Equipamento de Proteção Auditiva - EPA, devido a sua viabilidade, menos valor agregado, efetividade e fácil acesso (MEIRA et al., 2012). No entanto, as legislações vigentes e normalizações não garantem o uso de medidas regulares na prevenção do ruído (TAK; DAVIS; CALVERT, 2009; SONEGO; SANTOS-FILHA; 
MORAES, 2016). Atualmente muitas indústrias e segmentos produtivos não possuem uma gestão/regulamentação correta quando relacionadas ao sistema de segurança do trabalho de seus colaboradores (GANIME, 2010).

A Saúde Ocupacional - SO tem como objetivos: a promoção e manutenção do mais alto grau de bem-estar físico, mental e social dos trabalhadores em todas as operações; a prevenção entre os trabalhadores, de desvios de saúde causados pelas condições de trabalho; a proteção dos trabalhadores em seus empregos, dos riscos resultantes de fatores adversos a saúde; a colocação e manutenção do trabalhador adaptadas ás questões fisiológicas e psicológicas, em suma: a adaptação do trabalho ao homem e de cada homem a sua atividade (MENDES, 1980).

Segundo dados do Ministério da Previdência Social (2012), foram registrados 578 casos de trabalhadores que apresentaram PAIRO no ano de 2011 - no Brasil, certamente quase todos estes casos poderiam ser evitados se medidas preventivas tivessem sido tomadas a partir da quantificação deste agente físico. Com a finalidade de preservar a qualidade auditiva dos trabalhadores submetidos a níveis elevados de ruído, foi implantada a Norma Regulamentadora NR - 15, estabelecendo que o limite máximo de ruído contínuo ou intermitente é de $85(\mathrm{~dB})$ durante oito horas de trabalho (BRASIL, 2001a). Diante destas considerações o presente trabalho tem como objetivo avaliar a exposição de um colaborador ao agente ruído em uma empresa de reciclagem de plástico no município de Caçador/SC.

\section{MATERIAIS E MÉTODOS}

O presente trabalho desenvolve-se a análise da exposição ao ruído de um colaborador (função) em uma empresa que possui como o segmento de recuperação de resíduos classe IIB, no município de Caçador/SC, conforme Figura 1. No decorrer desse estudo, utilizou-se a metodologia descritiva com método dedutivo, utilizando a pesquisa documental de caráter exploratório por se caracterizar como pesquisa que parte do estudo geral para o específico (GIL,1991). 
Figura 1 - Localização da empresa do estudo

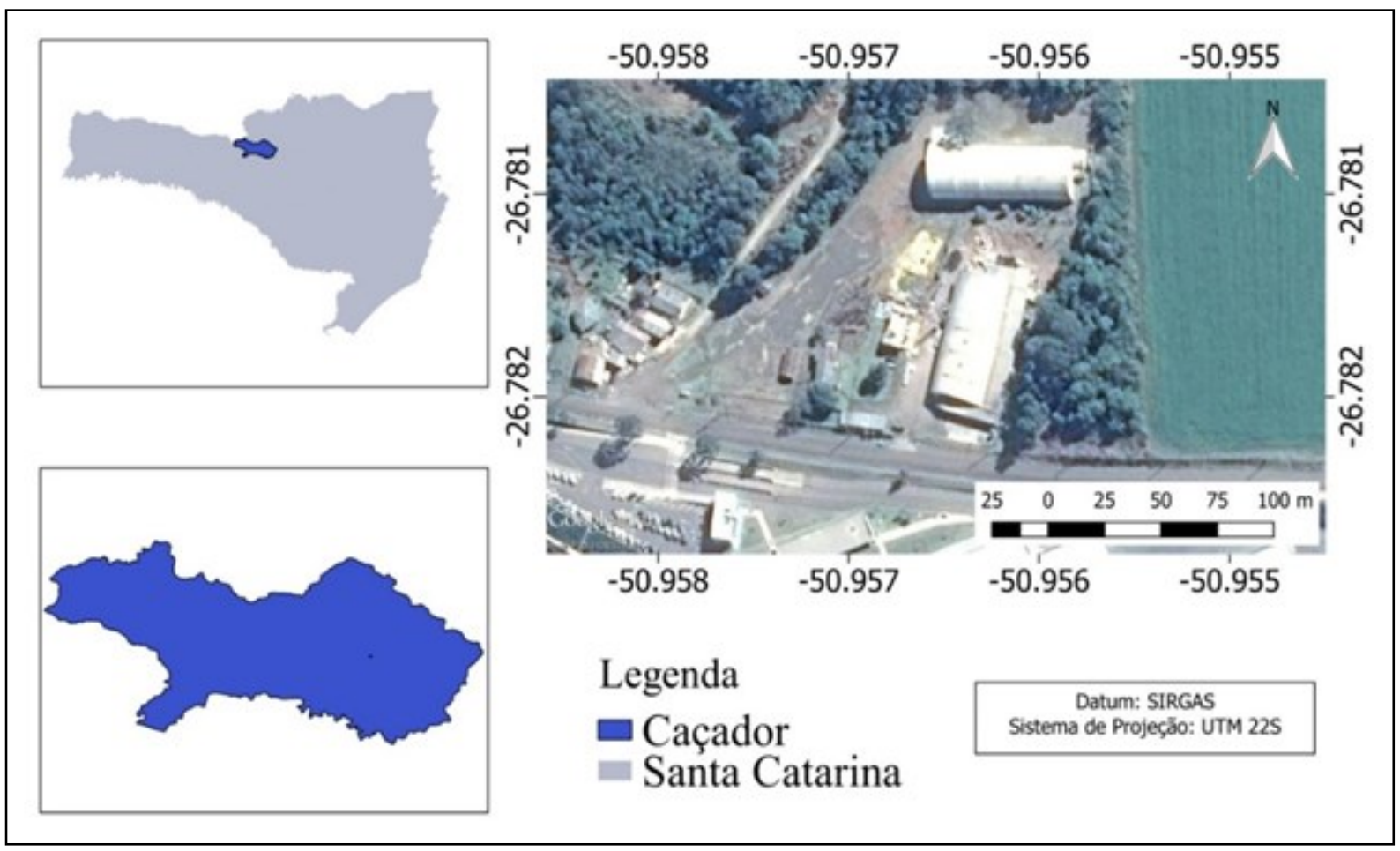

Para a realização da dosimetria do ruído utilizou-se o método da dose diária seguindo os critérios da Norma Regulamentadora NR-15 (BRASIL, 2001a), a qual estabelece os limites de tolerância para ruído contínuo ou intermitente durante a jornada de trabalho no Brasil. Para a análise da emissão de pressão sonora, utilizouse o procedimento metodológico disposto na NHO 01, a qual estipula o procedimento técnico para a avaliação da exposição ocupacional ao ruído (BRASIL, 2001b), através de um decibelímetro devidamente calibrado por um laboratório credenciado pelo INMETRO junto à Rede Brasileira de Calibração e à Rede Brasileira de Laboratórios de Ensaio.

Foram analisadas medições durante todo o horário de trabalho de um colaborador (manipulador do processo de extrusão do plástico) das 7:00 horas até as 16:30 horas durante um dia (8 horas) contabilizando medições conforme suas funções, compondo $100 \%$ da jornada de trabalho. Para a avaliação da emissão de pressão sonora foi utilizado as medições do nível sonoro equivalente $L A e q$, expresso em dB(A), conforme Formula 1 (ABNT 10151, 2000).

$L A e q=10 \log \frac{1}{n} \sum_{i=I}^{n} 10^{\frac{L i}{10}}$

Onde, $L i$ é o nível de pressão sonora, em $\mathrm{dB}(\mathrm{A})$, lido em resposta rápida (fast) a cada $5 \mathrm{~s}$, durante o tempo de medição do ruído; e $n$ é o número total de leituras. 
A dose de exposição foi realizada de acordo com NR- 15 e NHO-01, onde, se na jornada de trabalho ocorrer dois ou mais períodos de exposição ao ruído de diferentes níveis, devem ser considerado os seus efeitos combinados (BRASIL, 2001a; BRASIL, 2001b), conforme Formula 2.

$D=\frac{C 1}{T 1}+\frac{C 2}{T 2}+\frac{C 3}{T 3} \ldots+\frac{C n}{T n}$

Onde, D é dose de exposição ocupacional, $C n$ tempo total que um trabalhador fica exposto a um nível e Tn é o tempo máximo permitido de exposição a esse nível.

Os resultados foram expressos em médias logarítmicas das medições e serão comparadas com a Tabela 1. e Tabela 2., que apresentam o tempo máximo de exposição diária permissível para ruídos contínuos e intermitentes, estabelecido no anexo 1 (um) da Norma Regulamentadora NR-15 (BRASIL, 2001a) e na NHO-01 (BRASIL, 2001b).

Através do valor da dose (D) de ruído, pode-se calcular o Nível de Exposição de Ruído -NE, que é o nível médio representativo da exposição diária da NHO-01, conforme (Formula 3) (BRASIL, 2001b) e para calcular o NE da NR-15 (BRASIL, 2011a), seguirá a Formula 4.

$$
\begin{aligned}
& N E=10 \times \log \left[\left(\frac{480}{T E}\right) \times\left(\frac{D}{100}\right)\right]+85 \\
& L e q=\log \left[\left(\frac{\mathrm{D} * 8}{\mathrm{TE}}\right)+5,117\right] / 0,06
\end{aligned}
$$

Onde, TE é o tempo de exposição em minutos e o $D$ é a dose em porcentagem. 
Avaliação e caracterização de insalubridade pro exposição á ruído em uma empresa de reciclagem de plástico

Tabela 1 - Limite de tolerância para exposição do ruído conforme NR 15

\begin{tabular}{cccc}
\hline $\begin{array}{c}\text { NíVEL DE RUíDO } \\
\text { Leq } \mathbf{d B}(\mathbf{A})\end{array}$ & $\begin{array}{c}\text { MÁXIMA EXPOSIÇÃO } \\
\text { DIÁRIA PERMISSÍVEL }\end{array}$ & $\begin{array}{c}\text { NíVEL DE RUÍDO } \\
\text { Leq } \mathbf{d B}(\mathbf{A})\end{array}$ & $\begin{array}{c}\text { MÁXIMA EXPOSIÇÃO } \\
\text { DIÁRIA PERMISSíVEL }\end{array}$ \\
\hline 85 & 8 horas & 98 & 1 hora e 15 min \\
86 & 7 horas & 100 & 1 hora \\
87 & 6 horas & 102 & 45 minutos \\
88 & 5 horas & 104 & 35 minutos \\
89 & 4 horas e 30 min & 105 & 30 minutos \\
90 & 4 horas & 106 & 25 minutos \\
91 & 3 horas e 30 min & 108 & 20 minutos \\
92 & 3 horas & 110 & 15 minutos \\
93 & 2 horas e 40 min & 112 & 10 minutos \\
94 & 2 horas e 15 min & 114 & 8 minutos \\
95 & 2 horas & 115 & 7 minutos \\
96 & 1 hora e 45 min & & \\
\hline
\end{tabular}

Fonte: BRASIL, (2001a).

Tabela 2 - Limite de tolerância para exposição do ruído conforme NHO-01

\begin{tabular}{cccc}
\hline $\begin{array}{c}\text { NíVEL DE RUÍDO } \\
\text { Leq dB(A) }\end{array}$ & $\begin{array}{c}\text { MÁXIMA EXPOSIÇÃO } \\
\text { DIÁRIA PERMISSIIVEL }\end{array}$ & $\begin{array}{c}\text { NíVEL DE RUÍDO } \\
\text { Leq dB(A) }\end{array}$ & $\begin{array}{c}\text { MÁXIMA EXPOSIÇÃO } \\
\text { DIÁRIA PERMISSÍVEL }\end{array}$ \\
\hline 80 & $1.523,90$ minutos & 93 & 75,59 minutos \\
81 & $1.209,52$ minutos & 94 & 60,00 minutos \\
82 & 960,00 minutos & 95 & 47,69 minutos \\
83 & 761,95 minutos & 96 & 37,79 minutos \\
84 & 604,76 minutos & 97 & 30,00 minutos \\
85 & 480,00 minutos & 98 & 23,81 minutos \\
86 & 380,97 minutos & 99 & 18,89 minutos \\
87 & 302,38 minutos & 100 & 15,00 minutos \\
88 & 240,00 minutos & 105 & 1,72 minutos \\
89 & 190,48 minutos & 110 & 0,74 minutos \\
91 & 120,00 minutos & 113 & 0,46 minutos \\
\hline 2 & 95,24 minutos & 115 &
\end{tabular}

Fonte: BRASIL, (2001b).

Com a análise da dose de ruído os resultados foram posteriormente comparados com os apresentados na Tabela 3, a qual estabelece a atuação recomendada para cada tipo de exposição e as ações de controle. A análise busca diagnosticar a Higiene Ocupacional - HO do colaborador, com a determinação dos níveis de pressão sonora por faixa de frequência, permitindo ter o espectro sonoro do ruído da fonte analisada (Tabela 3), buscando a integração da NR 6 Equipamento de Proteção Individual - EPI, para o fornecimento da proteção adequada (BRASIL, 2011) e NR 15 - Atividades de Operação Insalubres - AOI, para atividades insalubres (BRASIL, 2001a). 
Tabela 3 - Interpretação e adequação dos resultados

\begin{tabular}{|c|c|c|c|}
\hline VALOR DA DOSE & $\begin{array}{l}\text { SITUAÇÃO DA } \\
\text { EXPOSIÇÃO }\end{array}$ & $\begin{array}{l}\text { CONSIDERAÇÃO } \\
\text { TECNICA DA } \\
\text { SITUAÇÃO }\end{array}$ & $\begin{array}{l}\text { NÍVEL DE ATUAÇÃO } \\
\text { RECOMENDADO } \\
\text { PARA AS AÇÕES DE } \\
\text { CONTROLE }\end{array}$ \\
\hline 0,1 a 0,5 & Aceitável & ------ & $\begin{array}{l}\text { Desejável, não } \\
\text { prioritária }\end{array}$ \\
\hline 0,6 a 0,8 & Aceitável & Atenção & De rotina \\
\hline 0,9 a 1,0 & $\begin{array}{c}\text { Temporariamente } \\
\text { aceitável }\end{array}$ & Séria & Preferencial \\
\hline 1,1 a 3,0 & Inaceitável & Crítica & Urgente \\
\hline Acima de 3,1 & Inaceitável & Emergência & Imediata \\
\hline $\begin{array}{l}\text { Qualquer, havendo } \\
\text { níveis individuais } \\
\text { acima de } 115 \mathrm{~dB}(\mathrm{~A})\end{array}$ & $\begin{array}{l}\text { Inaceitável, } \\
\text { recomenda-se } \\
\text { interromper a } \\
\text { exposição } \\
\end{array}$ & Emergência & Imediata \\
\hline
\end{tabular}

Fonte: SANTOS; SILVA (2000).

\section{RESULTADOS E DISCUSSÕES}

Durante o estudo pode-se observar que o colaborador possui a função de operação da câmera extrusora (Figura 2B), obtendo duas atividades, quais sejam: a) a introdução do plástico e água para a aglutinação do plástico (Figura 2C e 2D), e b) a função de coletar o material no pátio da empresa e levar até o aglutinador.

Figura 2 - Análise da atividade em estudo: Câmeras extrusoras (A); Local de trabalho do operado (B); Introdução de água na extrusora (C) e Introdução do plástico na extrusora (D)
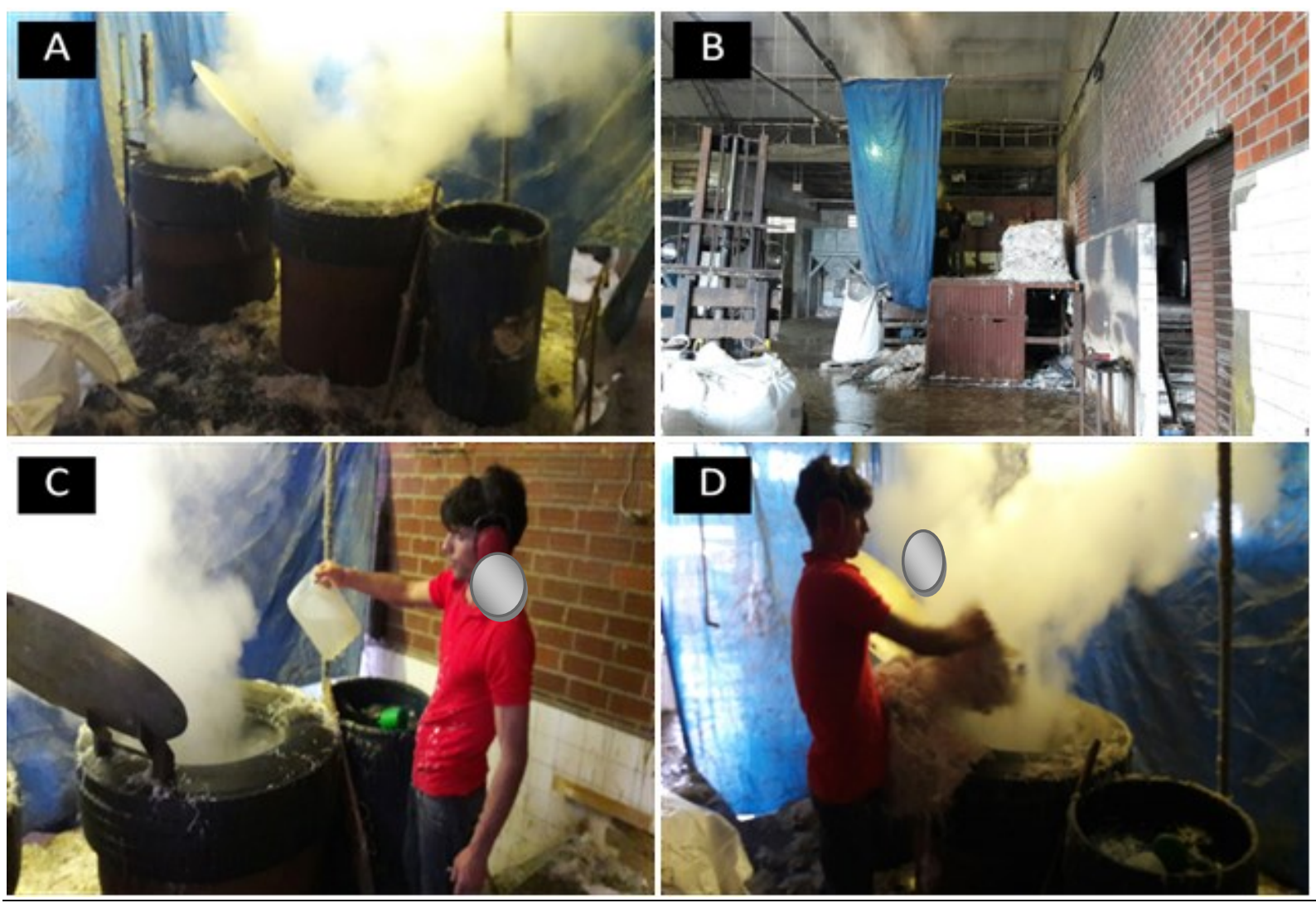

Saúde Meio Ambient. v. 8, p. 137-147, 2019 
Avaliação e caracterização de insalubridade pro exposição á ruído em uma empresa de reciclagem de plástico

A Tabela 4., apresenta os cálculos da dose, níveis de exposição e tempo permitido conforme NR-15 e NHO-01.

Tabela 4 - Medições e resultados

\begin{tabular}{|c|c|c|c|c|c|c|}
\hline NORMA & MEDIÇÃO & EQUIPAMENTO & $\begin{array}{l}\text { NIVEL } \\
\text { DE } \\
\text { RUÍDO } \\
\text { MÉDIO }\end{array}$ & $\begin{array}{c}\text { TEMPO } \\
\text { MÉDIO } \\
\text { ESTIMADO } \\
\text { DE } \\
\text { EXPOSIÇÃO } \\
(C n)\end{array}$ & $\begin{array}{c}\text { TEMPO } \\
\text { PERMITIDO } \\
\text { CONFORME } \\
\text { NÍVEL DE } \\
\text { RUÍDO (Tn) }\end{array}$ & $\begin{array}{l}\text { RELAÇÃO } \\
\text { Cn/Tn }\end{array}$ \\
\hline & 1 & Extr & 87,4 & 7 horas & $(86)$ & 1,17 \\
\hline NR-15 & 2 & Carregamento & 85,1 & $\begin{array}{l}1 \text { hora } \\
\text { Exposiç }\end{array}$ & $\begin{array}{l}1 \text { horas (100) } \\
\text { diária estimada }\end{array}$ & $\begin{array}{c}0,13 \\
C n / T n) 1,30\end{array}$ \\
\hline NHO-01 & $\begin{array}{l}1 \\
2\end{array}$ & $\begin{array}{c}\text { Extrusora } \\
\text { Carregamento }\end{array}$ & $\begin{array}{l}87,4 \\
85,1\end{array}$ & $\begin{array}{l}420 \text { Minutos } \\
60 \text { Minutos } \\
\text { Exposiç }\end{array}$ & $\begin{array}{l}302,38 \text { minutos } \\
480,00 \text { minutos } \\
\text { diária estimada }\end{array}$ & $\begin{array}{c}1,39 \\
0,13 \\
-C n / T n) 1,59\end{array}$ \\
\hline
\end{tabular}

Conforme análise durante 0 expediente de trabalho do colaborador, foi verificado dois níveis de ruídos.. Oprimeiro com $87,4 \mathrm{~dB}(\mathrm{~A})$ ( 7 horas - 428 minutos) referente ao processo de manipulação da extrusora, e o segundo referente ao carregamento do material para o processo apresentando $85,1 \mathrm{~dB}(\mathrm{~A})$ (1 hora -60 minutos).

Neste sentido, a Tabela 5., apresenta os pareceres quanto à classificação dos riscos para a atividade analisada, bem como as recomendações e ações, caso sejam consideradas.

Tabela 5 - Parecer sobre a função analisada

\begin{tabular}{cccccc}
\hline FUNÇÃO & $\begin{array}{c}\text { DOSE dB(A) } \\
\text { CALCULADO }\end{array}$ & NORMA & $\begin{array}{c}\text { AGENTE } \\
\text { RUÍDO }\end{array}$ & $\begin{array}{c}\text { CLASSIFICAÇÃO } \\
\text { DOS RISCOS }\end{array}$ & $\begin{array}{c}\text { NIVEL DE AÇÃO } \\
\text { RECOMENDADA } \\
\text { PARA AS AÇÕES } \\
\text { DE CONTROLE }\end{array}$ \\
\hline Operador & \multirow{2}{*}{87,18} & $\begin{array}{c}\text { NR-15 } \\
\text { NHO-01 }\end{array}$ & $\begin{array}{l}\text { Inaceitável } \\
\text { Inaceitável }\end{array}$ & $\begin{array}{c}\text { Critica } \\
\text { Crítica }\end{array}$ & $\begin{array}{c}\text { Urgente } \\
\text { Urgente }\end{array}$ \\
\hline
\end{tabular}

O estudo apresenta que durante a jornada de 8 horas o trabalhador estive exposto a um nível de pressão sonora equivalente a $87,18 \mathrm{~dB}(\mathrm{~A})$, acarreta insalubridade. Segundo Almeida (2000) quando expostos ao ruído os trabalhadores podem sofrer riscos, que podem interferir no seu trabalho, no cansaço e na comunicação entre os seres humanos, induzindo a perda auditiva induzida pelo ruído ocupacional, que está relacionada com o tempo de exposição, intensidade e susceptibilidade individual do trabalhador com o ruído.

Segundo a Brasil (2001b) deve ser adotado ações imediatas corretivas com valores iguais ou à cima de $85 \mathrm{~dB}(\mathrm{~A})$. Para a diminuição da exposição do colaborador a empresa forneceu o equipamento de proteção individual (Figura $2 \mathrm{C}$ e 
2D), Abafador Concha 3M Muffler (CA: 14235), atenuando o ruído de $87,18 \mathrm{~dB}(\mathrm{~A})$ para 73,18 dB(A) devido a capacidade de atenuação do ruído em $14 \mathrm{~dB}(\mathrm{~A})$. Diante do alto nível de ruído apresentado pela câmera extrusora, o profissional deve adotar medidas preventivas para evitar lesões auditivas, como também é necessário serem desenvolvidas novas pesquisas para verificar a redução do ruído na área industrial da empresa e, consequentemente, melhorar a qualidade de vida dos colaboradores (BRASIL, 2001b).

\section{CONCLUSÃO}

Com a realização da avaliação, conclui-se que a atividade é insalubre, com relação ao ruído. A avaliação constatou um nível de exposição de 87,18 dB(A), o que indica a necessidade urgente de algumas ações preventivas, que busquem evitar que este nível de pressão sonora afete a qualidade de vida do colaborador. Apesar das condições indicarem insalubridade, o colaborador da empresa do estudo utiliza protetor auriculares de inserção, ou tipo plug, com atenuação na faixa de 73,18 $\mathrm{dB}(\mathrm{A})$. A exposição ao ruído deve obter um monitorado pelo técnico responsável pela parte de segurança de trabalho das empresas, como também pela distribuição de EPI e cobrança da utilização do mesmo, sendo que esse é o primeiro mecanismo a ser utilizado para preservar a qualidade dos colaboradores das empresas e evitar futuros transtornos às empresas.

\section{REFERÊNCIAS}

ALMEIDA, S.I.; DE ALBERNAZ, P.L.; ZAIA, P.A.; XAVIER, O.G.; KARAZAWA, E.H.; História natural da perda auditiva ocupacional provocada por ruído. Rev Assoc Med Bras, 46(2):143-58; 2000.

ARAÚJO, S. A. Perda auditiva induzida pelo ruído em trabalhadores de metalúrgica. Revista Brasileira de Otorrinolaringologia, São Paulo, 68(1):47-52; jan./fev. 2002.

BRAGA, B.; HESPANHOL, I.; CONEJO, J.G.L.; MIERZWA, J.C.; BARROS, M.T.L.; SPENCER, M.; PORTO, M.; NUCCI, N.; JULIANO, N.; EIGER, S. Introdução à engenharia ambiental: $O$ desafio do desenvolvimento sustentável. 2. ed. São Paulo: Pearson Prentice Hall; 2005.

BRASIL. Ministério da Previdência Social. Anuário estatístico de acidentes de trabalho 2012. Disponível em: <http://www.previdencia.gov.br/arquivos/office/ 1_130108-164029-787>. Acesso em: 09 dez. 2016. 
BRASIL. Ministério do Trabalho e Emprego. Norma Regulamentadora NR-6 Equipamento de Proteção Individual - EPI. 68.ed. São Paulo: Editora Atlas; 2011.

BRASIL. Ministério do Trabalho e Emprego. Norma Regulamentadora № 15, de 28 de janeiro de 2011. Atividades e Operações Insalubres. Portaria SIT n. ${ }^{\circ} 203$; 2001a.

BRASIL, Ministério do Trabalho e Emprego. Norma de Higiene Ocupacional - NHO 01 - Avaliação da exposição ocupacional ao ruído, FUNDACENTRO; 2001b.

COELHO, J.M.; COELHO, R.A. Percepção de alunos e professores sobre a poluição sonora da Educação de Jovens e Adultos (EJA) em uma instituição pública de ensino. Revista Espacios, 36(23):E-2; 2015.

GANIME, J.F.; ALMEIDA DA SILVA, L.; ROBAZZI, M.L.C.C.; VALENZUELA SAUZO, S.; FALERIO, S.A. O ruído como um dos riscos ocupacionais: Uma revisão de literatura. Revista Enferméria Global. (19):1-15; jun. 2010.

GIL, A.C. Como elaborar projetos de pesquisa. São Paulo: Atlas; 1991.

LUSK, S. L.; HAGERTY, B. M.; GILLESPIE, B.; CARUSO, C. C. Chronic Effects of Workplace Noise on Blood Pressure and Heart Rate. Archives of Environmental Health, 57(4):273-281; jul/ago. 2002.

MENDES, R. Medicina do trabalho e doenças profissionais. São Paulo: Sarvier; 1980.

MELLO, J.G.A.; SANTOS, A.O. Avaliação quantitativa de ruído em uma empresa do segmento de informática no município de llhéus, Bahia. Revista Enciclopédia biosfera, 10(19):2466-2475; 2014.

MEIRA, T.C.; FERRITE, S.; CAVALCANTE, S.; CORREA, M. J. M. Exposição ao ruído ocupacional: reflexões a partir do campo da Saúde do Trabalhador. Revista de Saúde, Meio Ambiente e Sustentabilidade - InterfacEHS, 7(3):26-45; 2012.

SANTOS, C. A. C.; SILVA, P. R. P. Ruído Industrial - Causas e Efeitos em Indústrias Madeireiras. Curitiba; 2000. Monografia (Especialização em Engenharia de Segurança do Trabalho) Universidade Tecnológica Federal do Paraná.

SILVA, L. F.; MENDES, R. Exposição combinada entre ruído e vibração e seus efeitos sobre a audição de trabalhadores. Revista Saúde Pública, 39(1): 9-17; 2015.

SONEGO, M. T.; SANTOS-FILHA, V. A. V.; MORAES, A.B. Equipamentos de proteção individual auricular: avaliação da efetividade em trabalhadores expostos a ruído. Revista CEFAC, 18(3):667-676; 2016. 
TAK, S.; DAVIS, R.R.; CALVERT, G.M. Exposure to hazardous workplace noise and use of hearing protection devices among US workers-NHANES, 1999- 2004.

American Journal of Industrial Medicine, 52(5):358-371; 2009.

Artigo recebido em: 11/02/2019

Artigo aprovado em: 24/05/2019

Artigo publicado em: 18/06/2019 